Rabaska

Revue d'ethnologie de l'Amérique française

\title{
L'air ne fait pas la chanson
}

Une entrevue avec Conrad Laforte

\section{Jean-Pierre Pichette}

Numéro 1, 2003

URI : https://id.erudit.org/iderudit/201607ar

DOI : https://doi.org/10.7202/201607ar

Aller au sommaire du numéro

Éditeur(s)

Société québécoise d'ethnologie

ISSN

1703-7433 (imprimé)

1916-7350 (numérique)

Découvrir la revue

Citer ce document

Pichette, J.-P. (2003). L'air ne fait pas la chanson : une entrevue avec Conrad

Laforte. Rabaska, (1), 83-104. https://doi.org/10.7202/201607ar

Ce document est protégé par la loi sur le droit d'auteur. L'utilisation des services d'Érudit (y compris la reproduction) est assujettie à sa politique d'utilisation que vous pouvez consulter en ligne.

https://apropos.erudit.org/fr/usagers/politique-dutilisation/
Cet article est diffusé et préservé par Érudit.

Érudit est un consortium interuniversitaire sans but lucratif composé de l'Université de Montréal, l'Université Laval et l'Université du Québec à Montréal. Il a pour mission la promotion et la valorisation de la recherche. https://www.erudit.org/fr/ 


\title{
L'air ne fait pas la chanson
}

\author{
Une entrevue avec Conrad Laforte \\ Propos recueillis par Jean-Pierre Pichette ${ }^{1}$ \\ Université de Sudbury
}

\section{I - « JE SUIS L'ENFANT DE MON PÈRE », COMME DIT LA CHANSON}

\section{"C'est un docteur de l'Allemagne "}

Le nom de ma famille vient de Lefort. Mes ancêtres sont originaires de la Wallonie, mais ils vivaient en Allemagne. J'ai le baptistaire du premier qui est venu ici : Johannes Laurentius Lefort (1755-1814). Son père était clerc de notaire du clergé et il enseignait le français parce qu'il était Français. Mais il parlait plusieurs langues. Il avait marié une Allemande puis il enseignait à Burgen, diocèse de Trèves, sur la Moselle. Je suis allé visiter ce pays-là avec Roger Pinon. Puis je trouvais cet endroit très beau : je me demandais pourquoi il n'était pas resté là.

\section{«À Boston, il s'en est allé battre contre l'Anglais »}

$J$ 'ai trouvé la réponse dans l'ouvrage de Jean-Pierre Wilhelmy. À cette époquelà, au XVIII ${ }^{e}$ siècle, c'était au moment de l'indépendance américaine. L'Angleterre voulait reconquérir l'Amérique. Il fallait former une armée. Mais, si on envoyait des soldats anglais combattre des Anglais, ils ne voudraient pas s'entretuer. Le roi d'Angleterre a proposé aux petits rois d'Allemagne de les payer tant par tête pour chacun des hommes qu'ils leur enverraient. En Allemagne, les petits rois ne voulaient pas envoyer leurs sujets. Alors, quand il y avait des gens qui voyageaient et qui s'arrêtaient dans les hôtels, les soldats arrivaient le lendemain matin puis ils vidaient les hôtels : ils prenaient tous les gens qui venaient d'ailleurs puis ils les envoyaient en Angleterre. Je suppose que mon ancêtre a été pris comme ça. Mais, parce qu'il parlait le français, l'anglais, l'allemand, ils l'ont nommé commissionnaire du bureau de l'ordonnance.

1. Ce texte est un condensé de la généreuse entrevue que Conrad Laforte nous a accordée les 30 et 31 juillet 2002, à son domicile de Sainte-Foy, Québec. La transcription intégrale des enregistrements a été exécutée par Louis-Pierre Pichette (collection Jean-Pierre Pichette, enreg. 3748-3749; durée : 5h 30; 150 p. ms). 
Mon ancêtre est venu ici puis il s'est finalement installé à Québec. Son emploi était à la Citadelle, parce que c'était là qu'était toute l'administration. La famille s'était installée, à la suite du premier ancêtre, dans la région de Québec, surtout dans la basse ville. Son fils, Pierre Laforte (1808-1870), a eu tous ses enfants là.

\section{«Ah ! c'était un petit cordonnier »}

Mon grand-père s'appelait Alexandre Laforte (1854-1933) et c'était un cordonnier. Il avait marié Odile Paquet en 1879. À la fin de sa vie, il était venu rester chez nous. Il avait été contremaître dans une manufacture de chaussures à Québec. Quand il venait chez nous, il regardait nos souliers : «Viens me montrer tes chaussures ». Il regardait ça puis il disait : « Ça, c'est un tel qui a fait ça. Puis c'est bon ou c'est pas bon ». C'était son métier.

\section{«Écoutez la complainte, petits et grands »}

Il y a eu un grand feu à Saint-Sauveur à cette époque-là. Et toutes les maisons avaient brûlé, celle de mes grands-parents aussi. Après ce grand feu-là, ils ne savaient plus quoi faire. Ma grand-mère, Odile Paquet, a beaucoup pâti de cet incendie, parce qu'elle était enceinte et qu'ils n'avaient plus de maisons. Ils ont vécu dans une espèce de hangar, une remise ! Dans ce temps-là, on ne donnait pas de secours aux gens, sinon des montants ridicules. Elle avait fini par attraper la tuberculose puis elle est décédée à Saint-Sauveur en 1891.

Il n'y avait pas rien que mon grand-père qui était mal pris là-dedans. Il y a de nombreuses maisons qui ont brûlé : c'était un feu extraordinaire. Alors, pour s'occuper de ces gens-là, ils ont nommé des commissaires qui ont proposé de les relocaliser autour d'un lac, près du lac Saint-Jean. Il s'appelle encore aujourd'hui le lac des Commissaires. Mon grand-père, qui restait à Québec à ce moment-là, a acheté une terre puis il a été s'installer là avec ses enfants et sa femme. Comme c'était un cordonnier, il ne savait pas trop faire d'autres choses et il revenait à Québec travailler. Mon père restait là sur la terre pour travailler puis, quand l'hiver arrivait, avec mon oncle Odilon, il allait travailler dans les chantiers.

\section{"Qui t'a si bien instruit?"}

Mon père, Philippe Laforte (1887-1973) est né ici, à Québec, dans la basse ville, à Saint-Sauveur. C'était la quatrième génération de Laforte. Quand ils sont devenus orphelins, après la mort de leur mère, mon grand-père les a mis tous les deux, Odilon puis Philippe, à l'orphelinat, de l'autre côté du fleuve, à Lévis. Puis c'est là que mon père a été éduqué. C'était un type extraordinaire, mon père. Il était intelligent parce qu'il a appris l'anglais par lui-même comme ça. Il avait été à l'orphelinat, apparemment que c'était un endroit exceptionnel. 
François Brassard aussi a été au même orphelinat ; c'est là qu'il a appris la musique. C'était un bon orphelinat où l'on enseignait bien.

\section{«Au jardin de mon père »}

Kénogami, c'est un nom amérindien. William Price voulait avoir un beau nom pour sa ville. C'est lui qui a fondé la ville, mais tous les gens travaillaient à son usine de Kénogami. Là, il y avait une petite paroisse qui s'appelait déjà Kénogami. Alors il est allé voir le curé puis il lui a dit : "Changez votre nom ». Il lui a donné un montant d'argent et il a acheté le nom. Il a donné ce nom-là à sa ville. C'est la paroisse Sainte-Famille. C'était au début de la ville de Kénogami. On avait nommé un maire puis mon père avait été nommé promaire. C'est parce qu'il avait un petit peu plus d'instruction que les autres. À l'orphelinat, il avait appris beaucoup de choses. Ça l'aidait énormément. Et puis bien, des fois, il remplaçait le maire. Puis, à la fin de sa vie, il avait été nommé président de la Ligue du Sacré-Cœur. Quand il y avait des processions, il était dans le cortège en avant et tout le monde le suivait.

\section{«Dans les chantiers, il s'est engagé »}

Quand les Price ont fondé la compagnie de pulpe et papier, mon père et mon oncle sont venus à Kénogami puis ils se sont engagés. Comme c'était des gens de chantiers, ils ont travaillé à corder du bois. Kénogami, c'était l'endroit idéal pour le bois à cette époque-là, parce que le bois pouvait arriver des chantiers par les rivières. À un moment donné, la compagnie a eu besoin d'un conducteur de train, parce qu'elle possédait une voie ferrée pour sortir sa production et l'envoyer au chemin de fer qui était à Jonquière. Pour choisir le conducteur, on avait donné à chacun des candidats un petit livret à apprendre par cœur. Le jour de l'examen, on leur a enlevé les cahiers puis on leur a dit : «Maintenant, écrivez-nous tout ce qui était dans le livre ». Alors mon père a passé haut la main. Il a gagné puis il a été conducteur du train. C'est lui qui allait chercher les ordres des patrons qui étaient tous Anglais. Mon père savait peut-être un petit peu d'anglais au début, mais il l'a appris et il se débrouillait en anglais. Puis les patrons aussi savaient un peu de français et ils pouvaient se parler. Tous les jours, il allait prendre les ordres et faire son rapport de la veille. Alors, c'était un travail important et bien rémunéré. C'est un type extraordinaire, mon père. Ma mère aussi.

\section{«Vous aussi, ma bonne et tendre mère »}

Ma mère s'appelait Marie-Mathilda Dallaire (1895-1976), fille de LouisAllen Dallaire. Mon grand-père Dallaire avait un bateau, un caboteur, qui transportait des marchandises sur le fleuve et le Saguenay. À cette époquelà, le transport se faisait surtout par eau. Ma mère vient de l'Anse-Saint- 
Jean. Elle avait été un peu partout avec ses parents, parce que son père s'était engagé pour construire des chemins et il était habitué de commander beaucoup de monde. Quand mon père a connu ma mère, elle habitait à La Tuque. Elle travaillait dans un magasin de vêtements. Mon père, quand il a vu que son emploi avait été confirmé et qu'il était bien payé, il lui a écrit de venir le trouver. Elle est venue puis ils se sont mariés à Kénogami, le 11 août 1914, parce qu'il avait une position stable.

\section{«Mon père a fait bâtir maison »}

Alors, il s'est acheté un terrain puis il s'est construit une maison. C'est là que nous avons été élevés, nous autres. Ils ont eu douze enfants. On n'a jamais eu de misère, puis on n'a jamais crevé de faim, même durant la Crise. On aidait même des gens. Dans ce temps-là, nous avions souvent quelqu'un qui venait frapper à la porte à l'heure du repas.

\section{"Quand on a une grosse famille "}

Ma mère était une personne extraordinaire. C'est elle qui menait la maison. Mon père, lui, il travaillait, il venait à la maison pour manger et se coucher puis, après ça, il s'en allait travailler. Alors, c'est elle qui faisait tout. Et même, c'est quasiment une farce, c'est ma mère qui signait ses chèques. Il arrivait avec son chèque, il n'avait pas le temps d'aller à la banque, il le donnait à ma mère puis elle allait le signer. Un bon jour, mon père va à la banque pour changer son chèque. Il signe son nom : ils ont refusé de l'honorer, car ils ne reconnaissaient pas sa signature. Il a été obligé de retourner à la maison faire signer ma mère.

Puis une famille nombreuse, c'est une force. Les gens disent qu'avoir une grosse famille peut appauvrir. Mais c'est une force. Ma mère, une fois, avait envoyé ma sœur à la boucherie. Elle dit : «Tu vas demander qu'ils te coupent un morceau de viande de bœuf dans la surlonge. Puis après, tu lui diras : hachez-le ». Elle ne voulait pas avoir du steak haché qui avait déjà attendu deux jours de temps dans l'étalage. Puis elle dit : «S'il ne veut pas, tu lui diras qu'on va changer de boucher ». Le boucher a dit : " Vous auriez dû me le dire, que vous vouliez du steak haché, j'en ai dans le comptoir ». Ma sœur a répondu : « Ma mère m'a dit de faire cela et de vous dire que, si vous ne voulez pas le faire, on va changer de boucher ». Alors, là, il était choqué. Il a été voir son patron puis il est revenu, et il a fait exactement ce que ma mère avait dit. Il l'a fait parce qu'il n'avait pas les moyens de perdre quatorze bouches à nourrir. On était douze enfants puis, avec mon père et ma mère, ça faisait quatorze. C'est une anecdote qui montre un peu comment les gens pouvaient se débrouiller puis ce qu'on pouvait apprendre dans une famille. 
Mon père est décédé à 85 ans et ma mère, à 81 ans. Ils ont eu bien de la misère, mais, nous autres, nous n'en avons pas eu.

\section{"Je suis né par une journée d'automne »}

J'étais le cinquième. Je suis né le 10 novembre 1921. J'ai failli mourir avant même de venir au monde. Ç'a été épouvantable. Ma mère était enceinte de moi et puis mon père a eu un accident. Imaginez : si mon père était mort dans ce temps-là, il n'y avait pas de sécurité sociale —, elle aurait été mal prise avec ses enfants. Il a eu un accident de train : il avait été coincé entre un mur et un train. On pensait qu'il mourrait. C'est ma mère qui m'a conté ça. Puis les médecins ont dit : « Aujourd'hui, s'il passe cette nuit, il va vivre ». Là, bien, elle a eu peur de faire une fausse couche. Si mon père était mort, ç'aurait été terrible. Le lendemain, mon père avait passé la nuit puis il était encore bon. Il a guéri puis il a repris sa place sur le train.

"Ils m'envoyent à l'école... »

À Kénogami, il y avait deux écoles : l'école des frères du Sacré-Cœur pour les garçons et, l'autre, c'était le couvent des sœurs pour les filles. J'ai deux de mes sœurs qui ont été institutrices. L'école des garçons était d'un côté, l'école des filles de l'autre puis il y avait l'église dans le milieu. Je n'ai jamais été un premier de classe. Ma mère voulait que j'aille étudier au Petit Séminaire de Chicoutimi. Elle avait dans son idée que j'étais faible et elle voulait me protéger pour ne pas que je travaille à des travaux trop durs. J'y suis allé. J'ai fait tout mon cours classique là. Et c'est au petit séminaire que j'ai appris à écrire. Nous avions une heure de lecture tous les jours puis c'était bien organisé. C'était des prêtres séculiers. J'ai seulement de beaux souvenirs du Petit Séminaire de Chicoutimi. Pendant l'été, pour les vacances, je retournais à Kénogami parce que j'étais pensionnaire au séminaire.

\section{«Puis le foreman, c'est un gros boulé »}

L'été, j'allais travailler sur la pitoune. C'était dans un atelier de préparation du bois pour en faire de la pâte à papier. On appelait ça la woodroom. On pilait du bois. Tous les étés. Le contremaître, c'était un dénommé Castonguay ; c'était un colosse, une armoire à glace, une affaire épouvantable. Ce gars-là, il était fort ; il était fort puis il n'avait pas peur de personne.

Quand j'arrivais moi, pour venir travailler, aux premiers jours de juin, il était là, le matin, avec les gens qui attendaient l'heure pour commencer à travailler. À Kénogami, il y avait un entrepreneur de pompes funèbres qui s'appelait Crevier. Ça fait que, lui, quand il me voyait arriver là tout blême, devant eux autres qui étaient tous bronzés, il me disait : " Ah ! Crevier n'a pas voulu de toi cette année! Tu vas aller corder du bois ». Alors là, je ne 
disais pas un mot. Tout le monde riait. Ça, c'était sa farce. Il faisait ça tous les ans. Mais c'était un bon diable. Je n'ai jamais eu de misère avec lui. Puis, après ça, je m'en allais corder du bois. Moi, je choisissais rien que les petites bûches. Ça fait qu'il a commencé à m'expliquer que ce n'était pas plus difficile de prendre des grosses et que c'était bien plus d'avance. Il m'a montré comment faire. C'est vrai qu'on n'a pas besoin de forcer plus. C'était très facile avec le petit crochet qu'on avait.

\section{«C'est un brave gaillard»}

Il était habitué à moi parce que, le midi souvent, il arrivait pour aller changer les panneaux en haut, sur la chaîne qui conduit le bois sur la pile, pour qu'il aille plus loin. Si cela se brise, que ça s'emplit et bloque pendant que ça marche, il fallait arrêter tout le monde de travailler. Alors quand il pouvait, on les changeait pendant que tout le monde mangeait et que la chaîne était arrêtée. Il fallait s'arranger pour ne pas arrêter le travail. Ça, ça coûte cher à la compagnie. Alors, moi, je faisais ça pour l'aider. C'était des petits services que je lui rendais comme ça puis il avait de la reconnaissance. Parmi ces gens-là, qui travaillaient dur l'été puis qui s'en retournaient travailler dans les chantiers l'automne, il y avait des colosses. Certains voulaient se prendre avec Castonguay, mais ils n'en ont jamais été capables.

\section{"Au bord d'une fontaine, je me suis reposé »}

Il était aimable aussi. Il avait fallu que je fasse un travail de nettoyage difficile. Ça fait qu'il trouvait que ce n'était pas un endroit salubre parce qu'il y avait de l'eau. Il m'avait dit : «Bon, quand tu fais ton travail, tu nettoies tout ça puis, après ça, tu t'en vas faire un tour là de l'autre côté de la rue; puis cache-toi un peu et fais ce que tu veux. Repose-toi puis tu reviendras ". Moi, je n'avais rien à faire. Quand j'ai vu ça, je m'apportais un cahier. Puis, comme il y avait un concours littéraire à Radio-Canada, j'ai écrit mon scénario là, pendant l'été. J'allais me placer dans ce coin-là, à côté du Saguenay qui coulait en bas. Je prenais mon cahier puis j'écrivais, puis je mettais ça dans ma boîte à lunch. J'apportais ça à la maison. Le lendemain, je recommençais, j'allais là. Une fois, je ne m'en étais pas rendu compte, j'étais assis là comme ça et je travaillais puis Castonguay arrive avec le grand patron. Ils me voient et passent à côté de moi, comme s'ils ne m'avaient pas vu.

\section{« Jamais ne ferai folie d'abandonner mon troupeau »}

Le grand patron savait que j'étudiais. Un bon jour, il m'avait arrêté puis il avait pris la peine de parler français : il voulait me donner une promotion, me faire venir travailler dans un bureau. Puis il disait ça sérieusement. Ah ! 
je n'ai pas voulu. Ça ne m'intéressait pas, moi : j'aurais été pris à faire ça toute ma vie. J'avais trop d'ambition pour devenir un petit comptable. Puis j'ai refusé.

\section{"Que les amants sont souvent malheureux "}

J'étais en philosophie. Je voulais écrire du théâtre, mais seulement je ne savais pas trop comment faire. Il aurait fallu que je sois guidé pour ça. Parce que j'avais gagné ce prix-là, le deuxième prix du concours à la radio. Et j'avais été à Montréal rencontrer la personne qui dirigeait le concours, puis elle m'avait dit : « Vous avez le deuxième prix, celui des jurys intellectuels. Mais, elle dit, on a aussi l'appréciation du public là : c'est vous qui étiez le premier ». Ç'a été joué à la radio par des grands artistes, les meilleurs artistes. Il y avait Jean-Pierre Masson et des grandes vedettes comme ça. C'était en 1946. Ma pièce s'appelait «En pleine nature ». C'était une tragédie à propos de la cueillette des bleuets. Il y avait eu un feu à un endroit qu'on appelait le Grand Brûlé, et puis moi, quand j'étais jeune là, je cueillais des bleuets. J'aimais ça aller cueillir des bleuets. Puis, dans ce grand brûlé, il y avait des gens qui allaient cueillir des bleuets. Un des cueilleurs avait planté sa tente, pour pouvoir récolter les bleuets avec sa famille, et un autre avait pris sa place. Les deux hommes se sont chicanés et finalement l'un d'eux a mis le feu. Puis les enfants des opposants, le fils de l'un qui voulait marier la fille de l'autre, essayaient de calmer leurs pères : ce sont ces jeunes qui ont brûlé. Pour l'époque, ce n'était pas une belle morale. Je sais que les religieuses écoutaient ça, quand ç'a passé à la radio, et puis qu'elles ont fermé l'appareil. Fini.

\section{« J'ai pris mon écritoire »}

$\mathrm{Au}$ séminaire, j'ai écrit plusieurs pièces. Puis je les avais fait jouer par mes confrères. On en avait fait une sur Calixa Lavallée, à l'occasion du centenaire de sa naissance, en 1942. C'était tout un personnage, Calixa Lavallée, l'auteur de la musique de l'hymne national Ô Canada; ça fait que c'est moi qui avais écrit le texte pour le théâtre. J'avais donné mon texte et le directeur m'avait dit : « Bien, on aura probablement des choses à changer ». J'ai dit : «C'est correct $»$. Mais moi, j'étais là tout le temps quand il faisait les exercices. S'il voulait changer les choses, je lui expliquais. Ah bien, il ne le changeait pas. Ils ont joué ma pièce exactement comme je l'avais écrite. J'en ai écrit une autre pour un ancien missionnaire. J'avais de l'expérience. J'aurais pu continuer là-dedans, mais je me suis aperçu que ça me fatiguait ; des fois, ça me faisait passer des nuits blanches. Ça fait que je n'avais pas envie de me faire mourir à faire ça : j'ai arrêté. 


\section{« Je ne veux pas d'un avocat... "}

J'ai fait un an de droit à l'Université de Montréal. C'était intéressant au début. J'ai appris des tas de choses. Je me souviens encore de ces choses-là. Dans les questions de droit, je connais mon affaire. J'avais un de mes cousins qui m'avait prêté de l'argent pour que j'aille à Montréal faire mes études. Il avait un hôtel et un restaurant à Jonquière. Comme il voulait faire plus d'argent, il s'est acheté un hôtel à Montréal. Dans le contrat d'achat, c'était marqué qu'il y avait une servitude et qu'il ne pouvait pas posséder un hôtel dans son quartier. Mais il ne l'a pas compris. Là, je lui ai dit : « La prochaine fois que vous ferez un contrat de ce genre-là, prenez votre notaire à vous que vous payez. Lui, il va vous le dire. Autrement, vous vous faites avoir ». Il n'avait pas d'autre notaire que celui du vendeur : «Il ne m'a pas expliqué ça!» Bien, j'ai dit : « Vous comprenez bien qu'il n'était pas pour vous expliquer ça. S'il vous l'avait dit, vous n'auriez jamais acheté ». Les avocats c'est terrible!

\section{«Ils sont si malhonnêtes »}

Le droit, j'aurais trouvé ça intéressant ; mais, quand j'ai vu ce qui se passait, je n'aimais plus ça. Il y avait eu de la tricherie. Un groupe d'étudiants avait obtenu les questions puis ils avaient appris les réponses par cœur avant l'examen. Ça fait qu'ils ont tous passé ça, facilement. J'ai vu des gens qui arrivaient avec leurs notes de cours puis qui s'assoyaient dessus pour passer l'examen; de temps en temps, ils sortaient une feuille. C'était épouvantable, j'ai jamais vu tricher comme ça à l'université Laval. Là, je ne voulais plus continuer là-dedans. Alors j'ai arrêté puis, cet hiver-là, j'ai resté à Kénogami. Je me suis acheté une « dactylo » puis j'ai appris à dactylographier. Ç'a l'air fou, mais c'était très important de savoir dactylographier. Puis maintenant je peux dactylographier mes textes sans avoir besoin de regarder.

\section{"Regarde dans son gros livre, se mit à lire dedans »}

Ensuite, j'ai appris qu'il y avait des cours en bibliothéconomie et en bibliographie. Ça s'appelait l'École des bibliothécaires. Alors j'ai été à Montréal. Je me suis inscrit. Puis le cours a duré un an et demi. J'ai fait mon cours. J'avais eu le temps de me ramasser de l'argent parce que j'avais travaillé au printemps à Kénogami où on m'avait engagé de bonne heure. Là, j'avais fait un travail plus dur : c'était de ramasser les bûches dans la glace ou dans la neige et d'envoyer ça à la manufacture. Comme j'avais appris comment faire, ce n'était pas plus difficile. Ça me forçait les bras, ça me faisait de l'exercice en même temps. Quand les cours ont commencé, je suis allé étudier. C'est là que j'ai eu mon diplôme en bibliothéconomie de l'Université de Montréal (1949). À Québec, il ne se donnait pas de cours là-dedans. Ce que 
j'aimais le mieux, c'était la paléographie pour lire les manuscrits anciens. Aujourd'hui, quand j'ai des textes écrits à la main avec des plumes d'oie, je peux les lire. Ces études-là m'ont bien servi par la suite. Mais je suis retourné travailler à Kénogami, dans la pulpe, parce que je n'avais pas de position.

\section{II - S'ENGAgER POUR UNE ChaNSON}

\section{«Avez-vous besoin d'un engagé ? "}

À un moment donné, j'ai entendu dire qu'à l'université Laval on cherchait un bibliothécaire pour travailler en folklore. Un de mes confrères, qui connaissait bien $\mathrm{M}^{\mathrm{g}} \mathrm{S}$ Savard, avait appris qu'il cherchait quelqu'un pour classer les documents réunis par Luc Lacourcière et $\mathrm{M}^{\mathrm{gr}}$ Savard. Je suis allé voir $\mathrm{M}^{\mathrm{gr}}$ Savard après avoir travaillé tout l'été ; j'avais des sous dans mes poches : je pouvais attendre et faire ce que je voulais avant. Là, j'ai rencontré $\mathrm{M}^{\mathrm{gr}}$ Savard puis il a fait venir Luc Lacourcière. Ils voulaient m'engager. Alors $j$ 'ai dit : "Quand est-ce que je vais commencer?"- « Tout de suite, si vous voulez ». Bien, j'ai dit : « Là, il faut que je me trouve un logement et que j'aille chercher mes affaires à Kénogami ». C'est ce que j'ai fait. Je me suis trouvé une chambre et j'ai apporté ma valise puis tout ce que j'avais. C'était en novembre 1951. Quand j'ai commencé à travailler à l'université, je ne gagnais pas autant que ce que je gagnais à Kénogami. Les gens me disaient : «T'es bien fou, tu perds de l'argent ! » Moi, je savais que je voulais faire d'autres choses.

\section{«Ah! Ah ! l'homme engagé connaissait bien... »}

J'ai été bibliothécaire-archiviste aux Archives de folklore de novembre 1951 à 1975. On désirait quelqu'un pour faire de la classification parce que les documents augmentaient sans cesse : il fallait mettre de l'ordre là-dedans. Moi, j'arrivais, je connaissais tous les principes pour faire ça. Puis j'ai commencé à faire de la classification puis à acheter des livres. Je faisais toutes sortes de choses et c'est moi qui ai monté la bibliothèque.

On était dans les bâtiments du vieux Séminaire, rue de l'Université. On occupait le rez-de-chaussée. Il y avait Madeleine Doyon, qui avait une spécialité, elle aussi, puis elle préparait une thèse. C'est formidable ce qu'elle a fait sur les costumes. Moi, j'ai travaillé longtemps dans le même bureau qu'elle. Et puis je montais au bureau de la grande bibliothèque, la bibliothèque générale de l'université, pour classer mes livres. Un des prêtres du séminaire avait vu que toutes mes fiches étaient dans des boîtes de souliers : « Ç’a pas de bon sens, il faut un meuble pour ça!» Puis il m'a acheté un beau grand meuble avec des tiroirs puis tout. Alors c'était intéressant et plus facile. Quand les gens me téléphonaient pour savoir quelque chose, j'ouvrais mon tiroir puis je leur répondais tout de suite au téléphone. C'est moi qui classais tout : 
les coutumes, les chansons, les contes selon la classification d'AarneThompson.

\section{«Les maîtres qui m'enseignent... »}

$\mathrm{M}^{\mathrm{gr}}$ Savard m'avait dit : « Continuez d'étudier, ne faites pas rien que regarder le dos des livres et les classer. Lisez-les ». Puis je me suis inscrit aux cours de Lacourcière. J'ai suivi des cours de Marcel Trudel et de plusieurs autres professeurs. J'ai fait ma licence (1968), ensuite le diplôme d'études supérieures (1970) puis mon doctorat (1978).

Parmi les professeurs, $\mathrm{j}$ 'ai eu Marius Barbeau. Il venait passer une semaine ou quinze jours, puis il donnait sa série de cours. Il faisait ça d'une façon originale. C'était intéressant. Il était coloré. C'était un grand savant, $M$. Barbeau. J'ai suivi tous les cours qu'il a donnés jusqu'en 1958. Il a arrêté quand il a fait une attaque de paralysie cérébrale. C'est qu'il transcrivait ses contes qui étaient en sténographie. Il travaillait assis dans son lit puis, à un certain moment donné, il a eu cette attaque. Il était original. Il arrivait dans le cours avec des choses comme, par exemple, le fromage de l'île d'Orléans puis il nous faisait goûter à ça. Il nous mettait dans le sujet. Il apportait des exemples de toutes sortes et il abordait toutes sortes de sujets. Il avait étudié l'anthropologie en Angleterre. Puis il en a profité pour aller en France.

Marius Barbeau, je l'ai connu mieux à Ottawa. C'était entendu avec Lacourcière que j'irais là-bas pour faire l'inventaire des chansons du Musée national, surtout de la collection de Barbeau. J'y suis allé et il m'a très bien reçu. J'ai classé tous ses documents. C'est pour cela que j'ai beaucoup de références aux collections de Barbeau. Nous sommes allés enquêter ensemble, Lacourcière, Barbeau et moi, puis $\mathrm{M}^{\mathrm{gr}}$ Savard aussi je pense. C'était dans Charlevoix. Barbeau était un des grands maîtres. C'était un savant, Barbeau.

\section{«Va-t'en moine, tu trembles tout le temps »}

Un bon jour, il donnait un cours sur la chanson et la tradition orale. Et puis il arrive un personnage, qui s'assoit en arrière puis il écoute son cours. Quand le cours est fini, il suit $M$. Barbeau jusqu'à son bureau. Puis il entre dans son bureau et il lui donne un paquet de ses cahiers de La Bonne Chanson. M. Barbeau a pris le paquet et il l'a tiré dans sa poubelle devant lui. C'est Barbeau qui me l'a conté lui-même ! C'était l'abbé Gadbois qui lui avait remis ses «bonnes chansons ». Barbeau n'était pas d'accord avec ça. Il a pris des chansons que Barbeau avait publiées puis il a changé quelques notes et quelques mots, et voilà : c'est la bonne chanson. C'est mauvais ce qu'il a fait. Il changeait les paroles puis il changeait les notes. Et il a fait pareil pour des chansons qui étaient classiques : il a retouché les textes et les mélodies. C'est un commerçant. Puis il faisait de l'argent. Puis, comme il a fait beaucoup d'argent avec ça, il est considéré comme un héros. 


\section{«Ah! nous étions trois capitaines »}

En plus de Barbeau, la première équipe des Archives de folklore comprenait le fondateur, Luc Lacourcière, qui donnait des cours de littérature orale, l'abbé Félix-Antoine Savard, qui enseignait aussi en littérature, et Madeleine Doyon, première secrétaire puis professeur dans le champ des coutumes, du costume et des jeux. Il y avait parfois des invités. Roger Matton est arrivé en 1955 ; il donnait des cours sur la musique populaire et il a publié un recueil des chansons acadiennes recueillies par le docteur Dominique Gauthier. Ce qu'il a fait, comme transcription musicale dans cet ouvrage-là, c'est excellent. Il connaissait bien la musique. Il enseignait très bien aussi. Puis il y en avait d'autres qui venaient. $\mathrm{M}^{\mathrm{me}}$ Élisabeth Brandon travaillait à sa thèse et elle suivait des cours.

\section{«Car c'est ici que nous allons danser »}

Ensuite, il y a eu d'autres collègues qui sont encore vivants, comme Simonne Voyer qui est arrivée dans ces années-là aussi. Elle avait étudié à l'université, à New-York. Et puis, à la fin de l'année, on avait demandé à chaque étudiant de danser une danse de son pays. Et, arrivé à son tour, elle n'en savait pas. Elle n'a pas dansé. Quand elle est revenue, elle a dit : «Il faut que j'en trouve ». Elle a téléphoné aux Archives de folklore et on l'a mise en relation avec Madeleine Doyon qui était chargée d'enseigner la danse et elle l'a pilotée. Alors, toutes les deux, elles ont travaillé ensemble. Elle l'a emmenée faire des enquêtes puis Lacourcière aussi. Il lui fallait du répertoire et, dans les archives, on n'en avait pas beaucoup dans ce domaine-là. Tous, nous voulions l'aider le plus possible, parce qu'elle avait la technique et qu'elle pouvait étudier ces danses-là.

\section{«Monsieur le curé ne défend pas ça »}

Moi aussi, j'ai travaillé avec Simonne Voyer. Je l'avais emmenée voir mes informateurs et nous avions enregistré des danses. Ce n'était pas qu'une petite histoire. C'était à Baie-Sainte-Catherine. Un de mes confrères, qui était prêtre, était curé par là. Ça fait que j'arrive là puis je voulais qu'ils fassent une petite soirée de danses pour Simonne Voyer. Mon informateur ne voulait pas faire de fête ni danser des danses sans avoir d'abord obtenu la permission. Alors j'ai dit: « Tiens, on va aller voir le curé ». Ça fait que je vais voir le curé, mon confrère. Il me donne la permission, mais, en sortant de là, l'informateur dit : «C'est pas assez ça, le curé, j'aimerais mieux avoir la permission de $\mathrm{M}^{\mathrm{gr}}$ Dufour. » C'était le directeur du Séminaire de Chicoutimi et puis il avait une maison d'été là. Alors je suịs parti avec mon informateur puis on est allé là. $\mathrm{M}^{\mathrm{gr}}$ Dufour m'attendait parce que mon confrère l'avait déjà appelé. Ça fait que je lui explique ça, moi, devant mon informateur. 
$M^{\text {gr }}$ Dufour dit : « Pauvre monsieur, faites tout ce qu'il va vous demander, il dit, je vous donne la permission puis vous danserez telle danse », il a dit un nom de danse, et Simonne Voyer était là. Enfin, l'homme a invité tous les gens qu'il connaissait pour faire une belle grande soirée de danses. Ils ont dansé toute la soirée et puis Simonne Voyer enregistrait. Elle avait mis son magnétophone, son micro, quasiment à terre. C'était pour prendre les pieds. Moi, quand j'ai vu ça, j'ai pris le mien puis je l'ai mis à côté du violoneux pour prendre la musique. J'ai tout enregistré puis elle a fait de même de sorte qu'elle a pu disposer des deux documents.

\section{«Je m'en vas-t-à la chasse »}

Comme je n'avais pas d'automobile à ce moment-là, je me suis associé à Russell Young dans les premières enquêtes que j'ai faites. C'était en 1954,je pense. Lui, il faisait des enquêtes sur les chansons. Puis moi, je faisais des enquêtes sur le conte. Nous faisions équipe. Lui, il avait son magnétophone puis, moi, j'en avais un aussi. D'abord, la première fois, nous nous étions rendus à Kénogami, chez mes parents, et, de là, à l'Anse-Saint-Jean. Et c'est à l'Anse-Saint-Jean que j'ai commencé à recueillir des contes, chez John Lavoie puis chez madame Grégoire. Elle avait des beaux contes, elle, puis ses garçons jouaient du violon. C'était des bons vivants. Cette fois-là, nous avions été assez longtemps en enquête, à l'Anse-Saint-Jean puis au PetitSaguenay. J'ai rencontré à cette occasion mon informateur Jos Boudreault. Il était conteur dans les chantiers. Ce bonhomme-là était drôle puis il faisait des farces. Une fois, je lui avais demandé combien il avait d'enfants. Il m'a dit : «J'en aurais bien plus que ça si j'avais plus de femmes ».

\section{« Écoutez, je m'en vas vous conter »}

Puis à tous les endroits où j'allais, les gens me disaient: «Ah! si Ernest Gagné était là, lui, il en sait des contes ! C'est un bon conteur ! " Partout, ils me donnaient son nom. Souvent, je leur demandais où il demeurait et il n'y en a pas un qui le savait. À un moment donné, quelqu'un m'a dit: «Il est à Chicoutimi, dans la côte de la Réserve». De Kénogami, j'ai téléphoné à divers endroits en vain. Mais, en téléphonant à un magasin proche de là, le commis m'a dit qu'il connaissait un vieillard qui s'appelait comme ça. Et il me donne son adresse. Mon beau-frère est venu me conduire jusque-là. Là, dans la maison, je dis mon nom, ce que je faisais puis je demande à rencontrer monsieur Ernest Gagné parce que tout le monde me dit que c'était le meilleur conteur. Alors sa femme, elle se mit à rire puis elle dit : « Il est plus rien que bon qu'à ça, conter des contes. Mais, elle dit, c'est bien commode pour faire tenir les enfants tranquilles ». 


\section{"C'est la pure vérité !»}

C'était un conteur. Il contait des histoires et il en savait tout le temps des nouvelles. Lui, c'était un puits de contes ! Il ne racontait jamais les mêmes choses. C'était un type formidable. J'en ai recueilli plusieurs de lui. C'est lui qui contait les contes les plus longs. Ça durait à peu près trois heures. Lui, il contait dans les chantiers et ça c'était important. Ces conteurs-là, c'était des artistes. C'était quasiment une profession que de conter dans les chantiers. À ce moment-là, il n'y avait pas de télévision ni de radio dans les camps. Alors, ça reposait les bûcherons, qui étaient fatigués, d'entendre conter un conte. Il contait tous les soirs. Son conte, c'était comme une émission de télévision. Il commençait un soir, il racontait une heure de temps ; le lendemain, il continuait une heure de temps ; puis le troisième soir, il finissait. Il s'organisait pour que ça roule comme ça pendant tout l'hiver. Lui, ça ne le fatiguait pas, il pouvait conter des contes comme ça tous les soirs puis il ne manquait pas de contes : il en avait tout le temps. Je l'ai rencontré en 1954 et il avait 74 ans. Je suis retourné aussi le faire conter en 1955 et en 1956 . Il me donna 75 contes dont plusieurs duraient trois heures. Il connaissait plusieurs bon conteurs de ses amis. Il était alors trop vieux pour aller au chantier.

J'ai commencé au Saguenay puis après je suis allé à Chicoutimi puis au Lac-Saint-Jean, et puis j'en ai recueilli un peu partout. J'ai recueilli au delà de 600 contes. Je ne les ai pas tous publiés. Ensuite, je suis allé dans le comté de Bagot, à Saint-Théodore d'Acton, dans la famille de mon épouse, et j'ai rayonné un peu autour. J'ai rencontré de très bon conteurs, comme Delphis Daigneault qui m'a donné beaucoup de contes aussi et dont je prépare le manuscrit de son répertoire.

\section{« Je la cueillis feuille à feuille »}

Puis il y avait aussi des chanteurs. J'ai recueilli beaucoup de chansons aussi. En plus de mes 600 contes, ma collection, qui comporte un peu plus de 1000 enregistrements, est constituée de chansons, de morceaux de musique, de légendes et de toutes sortes d'autres choses. Mes enquêtes ont duré une dizaine d'années, à partir de 1954 jusqu'au moment où je suis allé la première fois en Europe, en 1964. Après ça, je n'ai pas fait beaucoup d'enquêtes parce que j'avais trop de documents à étudier. J'ai voulu faire mon terrain au Saguenay et au Lac-Saint-Jean parce que c'était un territoire encore vierge ; ailleurs, je serais arrivé dans les plates-bandes des autres, Barbeau (Charlevoix, Beauce), Lacourcière (Charlevoix, Gaspésie, Acadie), Gauthier (Acadie), et j'aurais recueilli les mêmes choses, ce qui aurait été moins intéressant. 


\section{"J'ai du plaisir ici... avec tous mes amis "}

Luc Lacourcière, $\mathrm{M}^{\mathrm{gr}}$ Savard et Marius Barbeau sont les chercheurs qui m'ont le plus inspiré pour mes travaux de terrain. Je les ai bien connus. J'ai travaillé avec eux. J'ai commencé à faire des recherches avec Lacourcière, dans Bellechasse, auprès du vieux Cléophas Fradette. On y allait toutes les semaines puis, lui, il nous attendait pour conter ses contes. Ça m'a appris un peu à voir comment on mancuvrait dans une enquête.

$J$ 'ai fait une enquête une fois avec $\mathrm{M}^{\mathrm{gr}}$ Savard et Luc Lacourcière, dans le rang de la Misère, à Baie-Saint-Paul. J'étais parti en excursion avec eux dans toute la région. Nous étions allés au chalet de $\mathrm{M}^{\mathrm{gr}}$ Savard. Pour y aller, il fallait prendre un bateau et c'est un de ses informateurs qui nous véhiculait et il contait des histoires et des légendes en traversant ! Nous avions visité le pays de Menaud et, en partant, j'avais un nom d'informateur. Nous étions allés le voir. Les gens de sa famille étaient en train de faire les foins. Mais le vieux, qui était assez âgé, est venu nous rencontrer. Puis là, il avait commencé à nous conter un conte. C'était « Jean de Bois ». J'ai tout enregistré. C'est un conte assez extraordinaire, rabelaisien. C'est un conte très long. J'étais avec $\mathrm{M}^{\mathrm{gr}}$ Savard puis Luc Lacourcière. Puis $\mathrm{M}^{\mathrm{gr}}$ Savard riait fort, puis le conteur aussi il riait tout le temps, puis moi je riais aussi. Moi, je riais moins fort parce que je m'occupais de l'enregistrer : c'était mon informateur puis c'est moi qui faisais l'enquête. Ah c'était agréable. Les relations avec Lacourcière puis Savard, l'équipe du début là, c'était convivial.

\section{«Là-haut, j’ai entendu la bergère Hélène »}

J'avais été photographié dans un journal, l'Action catholique du mercredi 16 février 1955, avec Dominique Gauthier, Luc Lacourcière, $M^{\mathrm{gr}}$ Parent, $\mathrm{M}^{\mathrm{gr}}$ Savard, Madeleine Doyon et le père Lemieux. Alors, Hélène Gauthier, quand elle a vu ça dans le journal, elle est venue me rencontrer au restaurant : j'allais toujours manger au même restaurant. Et c'est comme ça que l'on s'est connu. C'était en 1955, je me souviens. On s'est marié en 1957, deux ans après. Moi, je ne la connaissais pas. Mais seulement, quand elle m'a dit qu'elle était la nièce du docteur Gauthier, alors je n'étais pas pour lui dire : vat'en. Après ça, elle m'avait invité : il y avait des concerts là puis elle chantait. Elle avait même chanté à la radio une fois. Elle étudiait encore le chant. Elle partait de Charlesbourg, elle venait à Québec pour prendre des cours de chants. Elle avait conservé cette photo. En 1958, on a eu notre fille Esther.

\section{«En revenant des noces »}

Quand on s'est marié, on a fait comme tout le monde, on est allé en voyage de noces. On s'est en allé aux États-Unis, du côté de l'Atlantique, parce que tout le monde parlait d'Old-Orchard. On n'est pas resté longtemps là : ça 
faisait trop de bruit puis, sur la plage, il y avait des bouteilles cassées. On a rencontré un couple, découragé d'Old-Orchard aussi, et il nous a invité à embarquer dans sa voiture. Comme on était proche de Boston, on y est allé en autobus. Je suis allé à l'université de Harvard consulter une référence que Lacourcière m'avait donnée. C'est justement là que $j$ 'avais emmené Hélène avec moi. Alors, j'ai consulté cet ouvrage-là : c'était une thèse sur les chansons françaises au Canada. Puis ma femme, elle m'avait aidé à copier quelques références. Tous les documents que je cite, je les ai tous vus moi-même. Des fois, je les ai vus deux, trois fois.

\section{"...qui composait une chanson nouvelle»}

C'était la théorie du temps que les chansons avaient été composées par le peuple. Ce n'est pas plus le peuple qui les a composées que les écrivains. Bien sûr, comme elles étaient chantées par le peuple, elles étaient vraiment composées par le peuple aussi parce que les gens modifiaient les chansons. Il faut savoir que, avant 1850 environ, il n'y avait pas de droits d'auteur et que tout était anonyme. On ne sait pas qui a composé telle chanson. D'autres ont pu la modifier ensuite. Ça, c'est tout à fait sûr que ç'a fonctionné comme ça. Mais, quand les droits d'auteurs ont été reconnus, l'auteur et le compositeur donnaient leur nom. J'ai parlé de ce problème dans un article : "Chansons folkloriques, chefs-d'œuvre d'inconnus ».

\section{III - C'EST TOUJOURS LA MÊME CHANSON}

\section{«Silence, il va chanter »}

Et un jour, Luc Lacourcière m'arrive avec un dossier dans les mains. En parlant de ses recherches, il me dit: «Je me spécialise uniquement sur les contes puis, vous, vous allez vous spécialiser sur les chansons ». Et il me donne son dossier. Il y avait toutes ses notes qu'il avait prises à droite puis à gauche. Il avait un bon petit dossier. C'était bien bon : un bon petit commencement. Là je me suis spécialisé. C'est là que j'ai commencé à me spécialiser sur les chansons. Il ne voulait pas que je m'occupe d'autres chansons en dehors de celles recueillies en Amérique. Je me suis bien rendu compte qu'il fallait étudier aussi les chansons françaises d'Europe, de France, de Belgique et de Suisse. C'était difficile d'étudier l'Amérique française sans connaître au départ la chanson française : c'est la même tradition; c'est la même chose. Il n'était pas le seul à dire des choses comme ça : on étudiait le folklore de l'Amérique française. C'est pour cela qu'il disait que j'étais têtu. Je n'ai pas dit un mot puis j'ai commencé aussi à classer les pièces françaises. Puis, quand je voyais un livre de chansons ou un livre de contes d'ici ou d'Europe, je le faisais venir puis je le classais aussi. C'est comme ça que j'ai commencé mon catalogue. 


\section{"Dans la ville de Bytown »}

Et j'ai été faire l'inventaire des collections du Musée national, celle de Marius Barbeau, puis, après ça, j'ai fait toutes les collections des archives. J'ai été engagé par le Musée national. Monsieur Barbeau voulait que je devienne un employé permanent. Il a essayé, mais ça n'a pas marché. On a fait un concours, on avait mis toutes mes qualifications, mais moi j'ai spécifié une chose : je voulais être six mois à Ottawa et six mois à l'université Laval. Ç'a été refusé à l'université Laval puis à Ottawa. J'ai continué à faire ce que je faisais à l'université Laval. Je n'étais pas pour m'en aller m'exiler là-bas sans aucune possibilité d'avancement. J'ai inventorié la collection du père Anselme Chiasson, à Ottawa et ailleurs ; à la bibliothèque de Montréal, à la salle Gagnon, le fonds Massicotte, puis la collection du père Lemieux de Sudbury.

\section{«Mettez-vous donc à travailler »}

J'ai fait ces inventaires-là. C'était la première chose à faire. Autrement, comment peut-on classer des choses quand on ne sait pas ce qu'il y a dans le fonds ? Là, j'ai d'abord vu toutes les versions et plus d'une fois. Et c'est comme ça que j'ai commencé. Je me suis rendu compte de bien des choses, d'abord que les chansons n'étaient pas toutes faites pareil, mais qu'on faisait toujours des lois qui devaient s'appliquer à toutes les chansons. Alors ça ne marchait pas. En même temps, je me suis rendu compte qu'il fallait que je trouve une façon de classer les chansons - dans la première édition de mon catalogue, en 1958, j'ai tout expliqué ça - qu'il fallait distinguer les poétiques. C'est là que j'ai fait mes Poétiques de la chanson traditionnelle française. Mon inventaire a abouti à ça parce qu'on ne pouvait plus avancer si on ne le faisait pas. Les poètes de chaque siècle suivaient leurs règles qui étaient différentes de l'un à l'autre. Pour ces chansons-là, c'est pareil. Les laisses, c'est la technique du Moyen-Âge. Comment ça se fait que les gens la retiennent encore? C'est quelque chose d'intéressant. Dans les laisses, ce sont des assonances. Au treizième siècle, les poètes ont trouvé la rime. Il y avait une rivalité entre ceux qui utilisaient l'assonance et ceux qui préféraient la rime. Puis, pour discréditer la laisse et l'assonance, les rimeurs disaient aux autres qu'ils ne savaient pas écrire et qu'ils faisaient des « rimes en goret ».

\section{"Marchez tout doux, parlez tout bas »}

C'est à cause de ma position que monsieur Lacourcière me trouvait têtu. Un bon jour, il me présente le père Benoît Lacroix, qui est médiéviste. Je me suis dit : «Tiens, je vais lui parler des laisses ». Alors je commence à lui parler de ça. Il ne savait pas trop quoi me dire : "Méfiez-vous, monsieur Laforte, méfiez-vous parce que le Moyen-Âge, c'est tout un monde : sept siècles ». Ça m'a refroidi un peu. Et il n'avait pas l'air de me prendre au sérieux. Ça 
fait que, moi, je suis têtu, j'ai dit : « Je vais le prouver ». C'est là que j'ai fait ma thèse. Entre temps, je faisais des recherches à Paris puis Benoît Lacroix était en France aussi. J'étais là pour finir ma thèse. Il était à l'Université de Caen. Ça fait que je lui téléphone puis il m'a reçu. J'ai passé deux, trois jours peut-être. Il a lu toute ma thèse, qui était presque terminée. Après, il dit : «C'est évident ». Moi, ça me rassurait. Je n'avais pas fait ça pour rien. C'est comme ça qu'une amitié s'est développée entre nous deux. J'ai fait au moins deux communications avec lui. Il avait été de mon jury de thèse. Comme il avait douté au début, je voulais que ce soit irréfutable. Lacourcière aussi hésitait, quand j'ai commencé à écrire ma thèse. Avec le temps, quand il a vu tout le développement que ça donnait, il ne disait plus la même chose.

\section{"Tout de travers, tout à l'envers »}

Lacourcière, pour la chanson, convenait que je pouvais faire comme pour le conte. Mais pour la chanson, il n'y avait pas de catalogue. Patrice Coirault, en France, avait voulu faire un catalogue, mais il n'a pas été capable : c'était un autodidacte... Comment peut-on aller bien loin quand il manque la science de base. Moi, j'avais étudié la bibliographie, la classification. Alors, c'était mon domaine. C'est pour ça que j'ai pensé aux poétiques et que je les ai vues. $\mathrm{Si}$ on trouve une règle dans les chansons en laisse, on ne peut pas l'appliquer aux chansons strophiques. Ça ne s'applique pas parce que ce n'est pas pareil. Alors, moi, c'est là-dessus que j'ai travaillé.

\section{«Vous m'entendez bien »}

J'ai commencé par faire des inventaires puis j'étais toujours embêté parce que je me demandais comment faire, quel titre donner. Souvent je composais un titre puis je le changeais des fois parce que je me rendais compte après coup que ce n'était pas bon. J'en ai corrigé, mais j'en ai d'autres que je pourrais corriger encore ; et, si je continuais les études, je corrigerais les titres que Coirault employait. Pour que tout le monde puisse se comprendre, j'avais repris tous les titres des autres. Par exemple, celle que les gens d'ici appellent « le Rapide blanc » et que Coirault nomme « Le Moine tremblant et la dame »; je regrette d'avoir pris ce titre-là parce que ce n'est pas ça du tout. Je l'appellerais « Carnaval », parce que c'est une chanson de carnaval. Mais personne ne la comprend parce qu'elle a été transformée. Si on ne distingue pas les poétiques, on perd son temps. Il n'y a pas moyen de trouver autre chose. À partir de cette découverte-là, j'ai pu classer les chansons et puis les réunir.

\section{"En faisant mes emplettes dans la ville de Paris »}

Ce sont mes inventaires de chansons qui ont permis cela. Quand j'ai réussi à aller en Europe, là, ç'a commencé à compter. J'y ai passé toute l'année scolaire 
1964-1965. Ça me faisait toujours plaisir quand je trouvais des choses. À la Bibliothèque nationale, à Paris, il y avait quelqu'un qui voulait que je m'en aille. C'est que je faisais mes recherches dans tous les ouvrages de Coirault, parce qu'il n'a pas tout publié, et je les ai tous parcourus puis j'ai fait l'inventaire de tout son fonds. On n'a jamais voulu que je consulte son fichier de chansons, puis, pour me provoquer, dans la salle où j'allais toujours travailler, ils ont fait une petite exposition, vitrée, où je pouvais voir juste les fiches de Coirault. Je ne pouvais pas les consulter, c'était interdit. Il y avait une société d'amis de Coirault qui avait la main mise sur toutes ces choseslà. Je ne pouvais pas consulter son fichier personnel, mais ils ne pouvaient m'interdire de consulter sa collection de livres, parce que ce n'était pas lui qui les avait composés, ces ouvrages-là.

\section{«Allant de chambre en chambre »}

Après ça, je suis allé à la bibliothèque de l'Arsenal. C'est là qu'étaient tous les manuscrits de Victor Smith. Alors ça, j'en aurais eu pour un an à les examiner ; ça fait que $j$ 'ai fait faire le microfilm de toute la collection. Cette documentation m'a toujours servi et me sert encore parce que, même dans la préparation de mes contes scatologiques, j'ai trouvé une chanson scatologique du petit neveu de Richelieu. J'ai aussi consulté d'autres bibliothèques à Paris, celle du Musée des arts et traditions populaires, mais encore en province, à Rouen par exemple.

\section{«Vive la rose et le lilas »}

Au British Museum, j'ai trouvé un manuscrit très intéressant. J'avais découvert dans une publication des références au thème des couronnes de fleurs. Les couronnes de fleurs, c'est extrêmement important, pas rien qu'en France. Les Grecs avaient un marché aux couronnes de fleurs. Ça correspondait à nos chansons populaires où on fait allusion au langage des fleurs. Alors j'ai demandé au directeur du musée de voir le manuscrit cité. Il y avait, à ce moment-là, une exposition de beaux manuscrits et puis le livre en question était là. On l'a sorti de l'exposition pour me l'apporter. J'ai passé une heure ou deux à en faire l'inventaire. C'était des vies de saints et des choses sur la religion puis des coutumes et des chansons. J'ai trouvé là les illustrations que j'ai reproduites sur la couverture de mes Survivances médiévales. J'ai appris beaucoup de choses. C'est un manuscrit que personne n'avait vraiment consulté et qui était censé être incompréhensible parce que son titre est en anglais, mais il n'y pas d'anglais à l'intérieur. C'est du français et du latin. Ce volume était au nom de la reine d'Angleterre. Ça prenait rien que moi pour découvrir un tel manuscrit. 
IV - VOILÀ UNE AUTRE CHANSON

\section{"Devinez ce que j'ai trouvé "}

Après la publication des Poétiques, j'étais allé à Paris puis je tenais à rendre visite à Claudie Marcel-Dubois parce que c'était une spécialiste. Elle m'a dit tout de suite que je n'aurais pas dû parler des chansons de France, de Suisse et de Belgique, mais juste des chansons du Canada. Ensuite, elle m'a dit : " Bien, M. Laforte, vous avez fait un travail sur un sujet qui n'existe pas ; les poétiques du peuple, ça n'existe pas ». Elle me disait ça comme si elle était toute découragée et que j'avais perdu mon temps. Les poétiques, ça n'existait pas pour elle parce qu'elle était musicienne et que ça n'a pas rapport à la musique, mais aux paroles. Et alors sa compagne, Maguy Andral, est arrivée puis elle a commencé à dire le contraire de Claudie Marcel-Dubois : elle disait que ça existait, mais que c'était sur les paroles et qu'elles travaillaient sur la musique. Elles ne s'intéressaient pas aux paroles. Il faut voir dans ses travaux comment Marcel-Dubois a traité les textes : « Les paroles, m'avaitelle dit, ça ne me dérange pas, c'est la musique qui est intéressante ; le reste, on donne ça à des linguistes qui l'écrivent en écriture phonétique ». Tout de suite, là, elle se limitait à quelque chose puis elle ne pouvait pas aller plus loin. Tandis que, moi, c'est des ouvertures que je faisais. Puis ça va beaucoup plus loin parce qu'on arrive à quelque chose de certain. Puis elle n'est pas la seule à avoir dit des choses semblables.

\section{"Belle, embarquez dans mon joli navire »}

Au retour de mon premier séjour d'un an en Europe, on m'a demandé de faire un cours, en 1965, sur les chansons. J'ai toujours continué par la suite. Ils m'ont nommé chargé de cours, de 1965 à 1967, assistant de 1967 à 1973, puis titulaire. J'ai enseigné de 1967 à 1988, jusqu'à ma retraite. Je préférais la recherche. Je faisais de l'enseignement pour pouvoir faire de la recherche. Je n'ai jamais donné des cours en tirant ma matière des ouvrages des autres ; j'enseignais toujours d'après ma recherche à moi. J'ai aussi dirigé des thèses, celle de Simonne Voyer par exemple, dont j'ai été le codirecteur avec JeanClaude Dupont. C'est pour ça que je la connais bien.

Comme j'avais publié bien des ouvrages, que le catalogue était en chantier et qu'on avait commencé à le publier, il y a des étudiants qui voulaient préparer une thèse sur la chanson. J'ai surtout dirigé des mémoires de maîtrise. Entre autres : Madeleine Béland, sur « La Vie des voyageurs et des forestiers par leurs chansons » (1979) ; Georges Arsenault sur « Les Complaintes, mémoires d'événements tragiques survenus aux Acadiens de l'Île-du-Prince-Édouard » (1979) ; Carmen Roberge, sur la "Chanson traditionnelle à sujet religieux » (1985). Souvent la thèse qu'ils faisaient servait d'introduction et de 
présentation au corpus de chansons à l'étude. Il y a eu aussi Édith Champagne, qui a travaillé sur « Les Vêtements dans les chansons énumératives » (1979); Monique Jutras, sur les « Structures littéraires des chansons folkloriques à caractère épique » (1991); Laura Sadowsky a étudié le caractère identitaire de la chanson, en codirection avec Nancy Schmitz (1987) ; Lorraine Carrier a travaillé sur « La Chanson en laisse, rapport entre la structure mélodique et la formule strophique 101 » (1987); et Lise Bonenfant, «Étude comparée de la chanson "Par derrière chez ma tante" " (1979), en codirection avec Roger Matton.

\section{"Vive les matelots naviguant sur ces eaux !"}

J'ai surtout dirigé des femmes. Peut-être que les femmes me préféraient comme directeur, je ne sais pas. Souvent, c'était des étudiantes que j'engageais pour travailler au catalogue et qui, finalement, prenaient goût à la chanson. Pour faire le catalogue, c'était assez complexe et plein de petits détails ; les femmes ont plus de facilité dans les petits détails. Je m'en suis rendu compte seulement après coup : je n'avais embauché que des femmes pour travailler au catalogue. Oui. Et elles travaillaient bien.

\section{«En remontant la rivière »}

Parmi mes publications, c'est difficile de déterminer laquelle est la plus importante parce que, pour moi, tout a été marquant. D'abord, j'ai commencé en 1973 par La Chanson folklorique et les écrivains du XIXe siècle (en France et au Québec) pour montrer comment les écrivains ont considéré la chanson. Puis j'ai été obligé d'en faire une deuxième édition. C'est important parce que ce sont toujours les écrivains qui se sont intéressés aux chansons et que c'est dans leurs écrits que l'on retrouve les idées qu'ils avaient de la chanson; que ces idées soient bonnes ou non, il y a beaucoup de choses intéressantes dans leurs œuvres. Ils ont été les premiers à s'en occuper, mais ils représentaient, bien sûr, les théories de leur époque. Cet ouvrage a été très bien reçu.

\section{"J'en ai fait une flûte, un beau sifflet chantant »}

Ensuite, ce furent les Poétiques de la chanson traditionnelle française (1976) et le Catalogue de la chanson folklorique française (1977-1987). Mais, pour faire le catalogue, il a fallu que je fasse les poétiques. Avant, on croyait qu'une règle était valable pour toutes les chansons. $D$ 'autres disaient que les chansons n'obéissaient à aucune règle. C'est tout le contraire. Par exemple, les laisses. À partir du XIII ${ }^{\mathrm{e}}$ siècle, il n'y a pas un poète lettré qui a écrit ses vers selon cette structure. Puis le monde ordinaire continuait à les chanter puis à les écrire pareil. Les autres poétiques sont différentes. Alors, $j$ 'ai classé les versions sous un même titre et puis avec les références. Le premier catalogue 
que j'ai préparé, la version de 1958 et son supplément en 1964, servaient surtout à identifier les chansons. Je commençais et, à ce moment-là, je n'avais pas encore déterminé les poétiques. Ensuite, bien, j'ai fait un catalogue par poétique. Le catalogue ne donne pas le texte de la chanson, mais la référence aux textes. C'est un outil de recherche pour faire des études. Ce n'est pas une anthologie. Alors celui qui veut consulter les chansons, il va voir aux références et, par elles, il saura tout de suite où trouver toutes les chansons, avec les paroles et la musique la plupart du temps. Même dans les études que j'ai dirigées ou que j'ai publiées sur les thématiques des chansons, je n'ai pas choisi les plus belles pièces ni les meilleures, ni les plus connues. Certains m'en ont fait le reproche. Pourtant, comme je l'ai dit alors dans mon introduction, je voulais donner simplement des exemples. Si quelqu'un veut faire l'étude d'une chanson, il a toute la bibliographie que j'ai trouvée. Il faudrait qu'il y ait des études sur toutes les chansons. Ils vont continuer dans la même voie et puis, avec le temps, leurs études seront beaucoup plus intéressantes.

\section{« Je m'en vais en chantant le long du grand chemin »}

J'ai dirigé une série d'ouvrages, présentés d'abord comme des thèses, qui mettent en valeur des sections de mon catalogue. Ensuite, j'ai mené des études plus approfondies, comme mes Survivances médiévales dans la chanson folklorique (1981). Presque tout ce que j'écris est toujours appuyé par des documents d'enquêtes, des manuscrits ou des imprimés. J'ai retrouvé dans les manuscrits des chansons en laisse du XII ${ }^{\mathrm{e}}$ au XIX ${ }^{\mathrm{e}}$ siècle. Il est très difficile de dire qui les a composées. On a déjà avancé qu'elles avaient été composées par de mauvais poètes. Comment un mauvais poète peut-il produire un chef-d'œuvre? parce que beaucoup de ces chansons-là sont des petits chefs-d'œuvre. Aujourd'hui, il y a peu d'auteurs de chansons qui peuvent soutenir la comparaison avec ces chansons du Moyen-Âge, des chansons de tradition orale. La technique est tout à fait différente. Aujourd'hui, on expose son état d'âme. C'est : je t'aime, je t'aime, je t'aime vingt fois; puis : je te quitterai, je te quitterai, je te quitterai ; puis c'est tout. Dans les chansons en laisse, on raconte toujours une petite histoire.

\section{V - TOUT FINIT PAR DES CHANSONS}

\section{«J'ai quelque chose à dire»}

Je ne pouvais pas imaginer au départ ce que je ferais. Moi, quand je commençais une étude, je la commençais d'une façon méthodique, comme j'avais appris. Il fallait faire des inventaires et c'est en partant des inventaires que je me suis rendu compte de l'existence des poétiques des chansons. Moi, 
quand je lisais une chanson, il fallait que je comprenne. Quand je ne comprenais pas, je cherchais. Une chanson qu'on ne comprend pas, ce n'est pas intéressant. Mais, quand c'est écrit en dialecte, on ne comprend pas toujours. Les Français non plus, parce qu'ils ne savent plus les dialectes du Moyen-Âge. Au Moyen-Âge, chaque province avait son dialecte. Quand je suis arrivé dans les chansons qui parlaient des couronnes de fleurs, puis il y en a beaucoup, à force d'en voir, il fallait que je finisse par comprendre ce que ça voulait dire et puis d'où ça venait. C'est pour ça que j'ai fait une étude là-dessus. J'en ai fait d'abord une communication à Delphes.

\section{«Voici la fin de ma chanson »}

J'ai pris ma retraite un an avant le temps parce que ça me donnait plus de libèrté pour continuer d'écrire mes livres ou rééditer et compléter ceux que j'avais déjà écrits. Je pense que mes travaux, après ceux de Gagnon, de Barbeau et de Lacourcière, ont servi à mettre de l'ordre là-dedans. Pour pouvoir étudier les chansons, il faut qu'on voie l'ordre. Parce qu'il y a des textes dans les chansons. Ce n'est pas rien que de la musique. Et mettre de l'ordre, ça ne se fait pas dans une journée. Formé en bibliothéconomie et en bibliographie, j'ai pu me débrouiller dans toutes les classifications. C'est la Bibliothèque nationale à Paris qui avait la classification la plus archaïque. Mais il faut tout de même savoir se démêler dans tout cela.

\section{«Chantez-la sur un autre ton... vous le pouvez vous autres »}

Dans l'évolution de l'enseignement à Laval, le programme d'Ethnologie, autrefois les Archives de folklore, a été regroupé sous le département d'Histoire. Je pense que les historiens étaient plus proches de nous autres que les littéraires. Les littéraires voulaient nous englober, nous assimiler. En ce moment, c'est difficile de juger comment se trouve l'enseignement à Laval. Mais il n'y a plus personne qui donne des cours sur la chanson ; je me demande s'il y a encore des gens qui donnent des cours en ethnologie... Le retour des études ethnologiques viendra de France. L'autre jour, chez mon éditeur, il y avait un Français, un professeur, qui voulait me rencontrer. C'est que, en France, on me connaît plus qu'ici. Il y a des gens qui s'intéressent à la chanson là-bas. Puis ils consultent mes travaux puis ça évolue ; il y a des jeunes qui poussent puis on ne sait pas ce qui peut advenir. Mes travaux sont diffusés et les gens font comme on a fait, nous autres, avec les œuvres des gens des siècles passés : ils les regardent, ils les utilisent, ils prennent ce qu'il y a de bon puis, ce qui n'est pas bon, ils le laissent. 